\title{
El programa gubernamental Bienestar Digital y su efecto en estudiantes del nivel medio superior de la Zona Metropolitana de Mérida, México
}

\section{Rafael Ortiz-Pech*,Wendy Maribel Uc-May** \\ Y Rodolfo Canto-SÁenz***}

\section{RESUMEN}

Se investiga si el programa Bienestar Digital, implementado por el gobierno de Yucatán, cumplió su propósito, que consistió en otorgar una computadora personal a estudiantes del nivel medio superior con la condición de terminar satisfactoriamente sus estudios y postularse al nivel superior. Se realizó un muestreo de estudiantes beneficiarios en escuelas de la Zona Metropolitana de Mérida, Yucatán. Entre los resultados se muestra que el programa no cumplió con los objetivos, porque gran porcentaje de los beneficiarios reprobaron el curso, mientras que otros recursaron durante el ciclo escolar. De los estudiantes que cumplieron con sus estudios, se encontró que los equipos fueron usados para otros fines o que fallaron repentinamente. Se concluye la necesidad de replantear programas gubernamentales similares para el otorgamiento de computadoras y comprometer a los beneficiarios a cumplir con los objetivos esperados del programa.

Palabras clave: política pública, estudiantes, uso y aprovechamiento, bienestar social.

\section{Clasificación JEL: H52, I24, I25, I38.}

Profesor-investigador en la Facultad de Economía, Campus en Ciencias Sociales, EconómicoAdministrativo y Humanidades, Universidad Autónoma de Yucatán, México. Correo electrónico: rafael.ortiz@correo.uady.mx, orcid: 0000-0002-7049-5040.

** Tesista egresada de la Licenciatura en Economía de la Facultad de Economía, Campus en Ciencias Sociales, Económico-Administrativo y Humanidades, Universidad Autónoma de Yucatán, México. Correo electrónico: wendy.ucmay@gmail.com.

**** Profesor-investigador en la Facultad de Economía, Campus en Ciencias Sociales, EconómicoAdministrativo y Humanidades, Universidad Autónoma de Yucatán, México. Correo electrónico: rodolfo.canto@correo.uady.mx, orcid: 0000-0001-6812-4155. 


\section{ABSTRACT}

\section{The government program "Digital Welfare" and its effect on high school students in the Metropolitan Area of the city of Mérida.}

It is investigated whether the program known as "Digital Welfare" implemented by the Yucatan government fulfilled its purpose. The grant consisted of a personal computer to high school students under the condition of successfully concluding their studies and applying to college education. For this, a sample is made of beneficiary students within the metropolitan area of Merida, in Yucatan. Among the main results, it is shown that the program did not comply the objectives sincea large percentage of the beneficiaries failed the courses, while others repeated the scholar year. For students who completed their studies, it was found that the equipment was used for other purposes or that the device suddenly broke down. Among the conclusions reached, is to rethink similar government programs in the granting of computers, committing the beneficiaries to comply the expected objectives of the program.

Keywords:public policy, students, use and exploitation, social well-fare. JEL Classification: H52, I24, I25, I38.

\section{INTRODUCCIÓN}

Este trabajo surge de la necesidad de evaluar el impacto del programa de gobierno Plataforma Educativa de Bachillerato, conocido como Bienestar Digital (BD), que consistió en otorgar una computadora portátil a estudiantes del nivel medio superior con el propósito de que concluyeran su educación. El ciclo escolar considerado fue 2014-2017 y se realizó en la Zona Metropolitana de Mérida (ZMM), que incluye los municipios de Mérida y Umán, ambos en el estado de Yucatán.

La justificación para hacer esta investigación se basa, en primer lugar, en que este tema no se ha estudiado y en la indagación bibliográfica no se encontraron referencias sobre un programa similar; en segundo lugar, se desea saber si el programa logró cumplir sus objetivos. La importancia del trabajo radica en que este programa de gobierno tiene como meta que cada beneficiario concluya sus estudios y, de lograrlo, el estudiante obtendrá un perfil educativo superior a través de cono- 
cimientos y habilidades que le permitirán desempeñarse satisfactoriamente en el mercado laboral (INEE, 2011).

Aunado a lo anterior, el gobierno considera que si el beneficiario termina su educación media superior, le permitirá tener coherencia social, económica y política, lo que se traduce en costos sociales menores para el primero. Posteriormente, se transformará en una reducción del desempleo y pobreza extrema, a la vez que traerá una menor desigualdad social, mayor equidad de género, entre otros. Este argumento se fundamenta en lo que sostiene la Secretaría de Educación Pública: dotar a los estudiantes de una educación con competencias y conocimientos permanentes, que se materialice en una trayectoria laboral productiva (SEP, 2012).

En este sentido, los beneficiarios del programa gubernamental recibieron una computadora personal con la intención de que permanezcan en sus estudios y que adquieran conocimientos y habilidades al familiarizarse con una tecnología de información y comunicación (TIC), que puede impactar en su desarrollo profesional y educativo. Al proveer esa computadora, se cumple con lo que se declara en el artículo $3^{\circ}$ de la Constitución Política de los Estados Unidos Mexicanos: el derecho de todo ciudadano mexicano de recibir obligatoriamente educación básica (preescolar, primaria y secundaria) y educación media superior. Esta educación debe garantizarse con una infraestructura física, métodos de enseñanza, material educativo y docentes capacitados, de modo que sea de calidad.

A nivel estatal, el artículo 90 de la Constitución del Estado de Yucatán establece la obligatoriedad de la educación básica y media superior (Congreso del Estado de Yucatán, 2015). Esta norma se materializa cuando se compara la matrícula del nivel medio superior durante los ciclos escolares; por ejemplo, 73,651 estudiantes (ciclo 2012-2013); 79,182 estudiantes (ciclo 2013-2014); 81,058 estudiantes (ciclo 20142015) y 96,261 estudiantes (ciclo 2016-2017). Como se observa, en cada ciclo escolar la matrícula es creciente (INEE, 2018). Sin embargo, estas cifras no necesariamente reflejan permanencia en los niveles educativos, ya que la deserción escolar no está considerada. En este sentido, se sabe que durante el ciclo 2014-2015, la tasa de deserción estatal fue de $13.9 \%$, porcentaje alto en relación con el nacional, que es de $12.4 \%$ para el mismo ciclo. 
Ante este panorama desalentador, el gobierno del estado ha creado programas para disminuir el abandono escolar y aumentar la tasa de egreso, con el fin de estimular la permanencia estudiantil. En este tenor, el gobierno federal creó el programa Prospera, ${ }^{1}$ que otorgó becas económicas a los estudiantes; por su parte, el gobierno de Yucatán instauró el programa Plataforma Educativa del Bachillerato Bienestar Digital, que consistió en otorgar una computadora portátil a los estudiantes de educación media superior para que concluyeran este nivel educativo, cuya duración es de tres años (Gobierno del Estado de Yucatán, 2018).

El programa se desarrolló de acuerdo con el eje Educación de calidad, planteado en el Plan Estatal de Desarrollo 2012-2018 del Gobierno del Estado de Yucatán (2013). Su objetivo fue "incrementar la permanencia de los estudiantes en el nivel medio superior". Asimismo, el programa se implementó bajo el Programa Sectorial de Educación de Calidad 2013-2018 de la Secretaría de Educación Pública (SEP) para: "incrementar la eficiencia terminal del sistema educativo estatal". Para hacer el programa aplicable, se pusieron en marcha estrategias como: "Implementar el Programa de Bienestar Digital en la educación media superior que contribuya a reducir el abandono escolar y mejorar el desempeño de los alumnos" (Gobierno del Estado de Yucatán, 2017).

De este modo, el gobierno estatal creó en 2013 el programa Bienestar Digital para combatir el abandono escolar y aumentar la tasa de eficiencia terminal en el nivel medio superior. Por tanto, esta investigación toma una muestra de estudiantes beneficiarios de dicho programa en la Zona Metropolitana de Mérida (ZMM), que incluye la ciudad de Mérida y Umán, para averiguar si existió mejoría (impacto) en la eficiencia terminal de los estudiantes que ingresaron en el ciclo escolar 2014-2015 y que egresaron en el ciclo 2016-2017; es decir, dentro de los tres años idóneos para terminar el nivel medio superior (2014-2017). Con lo anterior se pudo conocer la trayectoria académica de los estudiantes durante los tres años de educación media superior (Secretaría de Gobernación, 2008).

El presente trabajo postula la hipótesis de que el programa Bienestar Digital tuvo una contribución positiva en la eficiencia terminal en los estudiantes del nivel medio superior y la computadora portátil

Este programa federal cambió de nombre en el sexenio 2018-2024 a Becas para el bienestar. 
coadyuvó a lograr este objetivo. Para desarrollar este trabajo, en el primer apartado se presenta la introducción; en el segundo apartado se encuentra el fundamento teórico sustentado en la teoría del capital humano y crecimiento económico, como una aproximación a la formación de capital humano, y el uso de tecnologías de la información y comunicación (TIC), que contribuyan a la economía; en el tercer apartado se incluye evidencia empírica, donde se muestra la importancia de la educación y el uso de tecnologías de la información y comunicaciones. En el cuarto apartado se presenta la metodología de esta investigación; posteriormente se presentan los resultados del trabajo. Para finalizar, se presentan las conclusiones y recomendaciones.

\section{TEORÍA Y EVIDENCIA EMPÍRICA}

\subsection{La formación de capital humano y crecimiento económico}

La formación de capital humano se entiende como el recibimiento de educación e incluye la adquisición de conocimientos, experiencia y entrenamiento laboral. Todo este proceso se genera, en gran parte, gracias a la educación formal; es decir, no se produce repentinamente. Cardona et al. (2007) indican que el capital humano involucra conocimientos, capacitación, experiencia y condiciones de salud de una persona, entre otros factores que se transforman en capacidades y habilidades para hacerlo productivo y competitivo dentro de la actividad económica en que se desempeña. Por su parte, Navarro (2005) define la formación de capital humano como el valor que generan las capacidades y habilidades del individuo mediante la educación, la capacidad de conocer, perfeccionarse, tomar decisiones acertadas y relacionarse con los demás.

De esta manera, el capital humano se convierte en un determinante del crecimiento económico por medio de la educación, ya que contribuye al avance de los conocimientos con mejoras cualitativas en la fuerza de trabajo. En este caso, la rentabilidad del dinero invertido en recursos humanos es más grande que la rentabilidad en capital físico. Por tanto, la educación no debería considerarse como una actividad de consumo, sino como inversión, pues obtiene tasas de retorno más altas comparables con el capital físico (Cardona et al., 2007). 
A su vez, Romer (1990) indica que el factor clave para la mejora económica se explica por el capital humano en la escuela; esto es, el desarrollo de la fuerza de trabajo efectiva es resultado de las nuevas tecnologías educativas. De este modo, el conocimiento es el factor de producción más importante, ya que es capaz de incrementar la productividad marginal, pues las empresas que contratan y acceden a mejor personal mejorarán su productividad. El modelo de Romer considera una función de producción con rendimientos constantes o crecientes, con factores productivos acumulables, que derivan en la siguiente función $Y=F(K, L, N, H, A)$; donde, $Y$ : Producción, $K$ : Capital, $L$ : Fuerza de trabajo, $N$ : Tierra, $H$ : Capital humano, y $A$ : Tecnología (Bravo, 2013).

Este modelo se basa en tres premisas: a) la acumulación de capital y el cambio tecnológico en conjunto, que incrementan el producto por hora trabajada; b) nuevos conocimientos se traducen en bienes con valor práctico, y c) el desarrollo de nuevas y mejores instrucciones equivalen a definir la tecnología.

Aunado a lo anterior, Lucas (1988) sostiene que la formación de capital humano se valora por la acumulación de conocimientos; existen dos tipos: voluntario e involuntario. El primero tiene que ver con asistir a la escuela y adquirir conocimientos; el segundo consiste en aprenderhaciendo; es decir, la teoría y la práctica. De esta forma se obtiene un mejor nivel de educación, toda vez que la formación educativa de cada persona aumenta el capital humano, el cual contribuye a un mejoramiento de la productividad de la economía por medio del spill-over (efectos secundarios) del conocimiento. De igual modo, si un estudiante en formación de capital humano se rodea de otras personas con alto nivel de capital humano (profesores o especialistas en un área de trabajo), puede recibir una externalidad positiva, de modo que se espera sea más productivo.

\subsection{La formación de capital humano y el uso de las tecnologías de información y comunicaciones (TIC)}

La implementación de las TIC en las aulas se ha incrementado de manera acelerada mundialmente y su incorporación no se limita únicamente a proporcionar equipos y programas, sino también a construir un uso educativo y didáctico de las mismas. Esto trae como resultado que los usuarios (estudiantes) accedan a la información de la red para realizar actividades sencillas o complejas y desarrollen procesos cogni- 
tivos; no obstante, puede ser que los docentes no estén capacitados o actualizados para el uso de estas tecnologías, lo cual es una desventaja respecto a los estudiantes (Díaz-Barriga, 2013).

Igualmente, el uso de las TIC puede acrecentar las desigualdades, dado que sólo la población con capacidad económica puede adquirirlas y usarlas, ya sea dentro de la formación de capital humano o dentro del mercado laboral; en contraste con los marginados que habitan en zonas apartadas y que no tienen acceso a estas tecnologías. De la misma manera, la formación de los estudiantes como capital humano, que usan las TIC, puede afectarse cuando se convierte en distractor o entretenimiento.

Por ello, Aguilar (2016) señala que las escuelas de alto estatus socioeconómico que usan TIC profundizan en las habilidades de entendimiento, mientras que las de bajo estatus se concentran en la familiarización del equipo. También menciona que la brecha digital ya no se reduce al equipo y su conectividad, sino al desarrollo de habilidades $\mathrm{y}$ valores para construir aprendizajes que propicien la inclusión y reduzcan las desigualdades existentes.

De esta manera, si un estudiante en formación de capital humano usa esta tecnología, puede ser capaz de generar conocimientos o diseños, y contribuir al proceso de innovación, lo cual se traducirá en producción. Sin embargo, este proceso no es obligatorio; el estudiante puede decidir dedicar su tiempo a trabajar o seguir adquiriendo educación formal. Su decisión tiene un incentivo económico, un salario pagado que crece en proporción con su acervo de capital humano acumulado (Ocegueda et al., 2013).

En general, la integración de las TIC a los sistemas educativos es compleja, pues depende de factores como la infraestructura, competencias de los docentes y los educandos, métodos de enseñanza, disponibilidad institucional, planes de estudio, disponibilidad de recursos financieros, entre otros. De esta manera, el problema con las TIC radica en que muchos países en desarrollo carecen de suficientes computadoras, y en otros casos no cuentan con internet o éste tiene mala calidad. Lo peor ocurre cuando en algunos países el sistema de suministro eléctrico falla demasiado o está ausente como servicio, de modo que las TIC no funcionan (Unesco, 2015).

Con base en el argumento anterior, la OECD (2015) indica que los estudiantes carecen considerablemente de capacidad para usar la 
computadora para asuntos escolares pues, aunque se usan en el salón de clase, su impacto en lo académico no es el mejor. Para quienes la usan de manera moderada, su rendimiento educativo tiende a ser mejor en comparación de quienes la utilizan ocasionalmente en su formación. Sin embargo, los resultados académicos de los estudiantes que la usan frecuentemente en la escuela pueden ser peores, si se tiene en cuenta inclusive su origen social.

En este sentido, la Unesco (2009) reconoce que el uso de las TIC en la educación amplía las oportunidades de aprendizaje y mejora la calidad de la educación al incorporar métodos avanzados de enseñanza; pero aclara que, a pesar de los avances en su uso, no hay evidencia empírica de que tenga beneficios en los usuarios, puesto que no hay datos que sustenten su impacto real, de modo que todos los argumentos son ambiguos o, en el mejor de los casos, discutibles.

\subsection{Evidencia empírica}

La revisión bibliográfica no permite encontrar estudios específicos sobre computadoras y educación; no obstante, la investigación clásica de Romer, Mankiw y Weil (1992) muestra cómo el nivel educativo influye en la economía; para ello consideran el número de años invertidos en la formación de capital humano y el crecimiento económico. Los resultados indican que tienen una correlación con sentido positivo y, de hecho, contribuyen a una aceleración en la convergencia condicional (formación de capital humano), pues demostraron que los países analizados para el periodo 1960-1985 tienen menor dispersión del ingreso.

Por su parte, Ocegueda et al. (2013) muestran cómo la educación impacta en la economía por medio de la inversión en el capital humano, hecho que permite salir de la trampa de la pobreza persistente. Para acelerar este proceso, es necesario impulsar el capital físico junto con más nivel tecnológico; el papel del gobierno es crucial con la estimulación del crecimiento, ya sea invirtiendo más en progreso tecnológico o incidiendo en el sector productor de tecnología.

Para demostrar este argumento, se consideran todos los estados mexicanos por su nivel escolar (desde primaria hasta posgrado) como variable proxy de la formación de capital humano, y se relacionan con la inversión según el valor agregado bruto para el periodo 1990-2008. Los resultados muestran que el nivel bachillerato no fue estadísticamente significativo, pero sí presenta un sentido positivo; es decir, habrá mayor 
inversión en producción, así como mayor cantidad de jóvenes en este nivel. También demuestran la existencia de una relación positiva entre ingresos bajos-medios y el nivel medio superior, aunque el resultado no fue significativo; se puede decir que un trabajador recibe más ingresos si cuenta con el nivel medio superior (comparado con niveles educativos inferiores). Asimismo, sostienen que la inversión en educación impacta en la economía, pues se esperan mayores niveles de ingresos.

En el mismo sentido, Galvez-Soriano (2020), con base en el modelo de Uzama-Lucas, subraya la importancia de la educación para la economía de México. Entre sus hallazgos postula que un aumento de $1 \%$ en la formación de capital humano provoca un aumento de $1.59 \%$ de la producción nacional. También sostiene que un aumento de $1 \%$ en años de educación, contribuye a $2.2 \%$ de incremento de la tasa de crecimiento del PIB. Por su parte, Villareal (2016) estudió la relación entre años de escolaridad y los ingresos recibidos en México para el periodo 1992-2001; concluye que existe una relación positiva, ya que al estudiar un nivel educativo adicional, el ingreso se incrementa entre 5.7 y 10 por ciento. Este resultado lo contrasta para el periodo $2000-2010$ y llega al mismo resultado. Por tanto, concluye que se requiere evitar que los jóvenes abandonen su formación de capital humano.

Referente a la evaluación de programas sociales a nivel nacional, el Coneval (2020b) publicó los resultados de evaluaciones dentro de la Ley Federal de Transparencia y Acceso a la Información Pública Gubernamental. Las evaluaciones incorporan información desde 2007 e incluyen el diagnóstico, diseño, estrategia, impacto, entre otros elementos. Según esta institución, se han hecho 2,800 evaluaciones a programas y políticas de desarrollo social en México, de las que sobresalen: "Evaluación de diseño con trabajo de campo del programa beca universal para estudiantes de educación media superior Benito Juárez (2019-2020)"; "Evaluación de diseño con trabajo de campo del programa jóvenes escribiendo el futuro 2019-2020"; "Evaluación del programa servicios de educación media superior 2019"; "Evaluación de consistencia y resultados del programa expansión de la educación media superior y superior 2017"; "Evaluación del programa de inclusión digital 2017" y "Evaluación del programa sectorial de educación 2016". 
A nivel estatal, el Coneval (2020c) evalúa y monitorea programas de Yucatán con el objetivo de contribuir a una mejora de la gestión gubernamental. Para ello se publican los documentos de evaluación, incluyendo los avances, áreas de oportunidad y retos para el estado. El Coneval indica que se presenta la información por transparencia de las políticas públicas, así como enlaces a los programas sociales. Por tanto, Yucatán es una entidad clasificada con un alto nivel de evaluación y la evidencia afirma que entre 2011 y 2019 la puntuación de diagnóstico fue de $51.8 \%, 11.4 \%$ mayor a la media nacional. En relación con el componente normativo, las mejoras son constantes; lo mismo para el componente práctico.

\section{Metodología}

La investigación realizada en 2017 fue cualitativa y cuantitativa; para el primer caso se obtuvo información de los estudiantes por medio de un cuestionario (fuente primaria) con preguntas abiertas. Para el cuantitativo se utilizaron los datos generados por el cuestionario, a partir del cual se realizó un análisis estadístico en el que se relacionaron las variables de interés. Los datos obtenidos fueron generados por los beneficiarios del programa Bienestar Digital, estudiantes del sexto semestre de nivel medio superior (bachillerato) en los municipios de Mérida y Umán, de la ZMM. Como fuentes secundarias se utilizaron datos abiertos de la Secretaría de Educación del Estado de Yucatán (Gobierno del Estado de Yucatán, 2017) y de la Secretaría de Educación Pública (SEP, 2013; 2014; 2015).

\subsection{La muestra}

El cuestionario se aplicó a beneficiarios del programa Bienestar digital que ingresaron al nivel medio superior en el ciclo escolar 2014-2015 y egresaron en el ciclo escolar 2016-2017. La información sobre la población beneficiaria fue proporcionada por la Secretaría de Educación del Estado de Yucatán; se tomó una muestra de los municipios de Mérida y Umán, pertenecientes a la ZMM.

La población total de beneficiarios del programa fue de 4,326 estudiantes de los municipios de Mérida y Umán, de acuerdo con la plataforma Bienestar digital. Al principio de la investigación se planteó 
realizar la técnica de muestreo aleatorio simple, ${ }^{2}$ que se consideraba tenía un nivel de confianza de $95 \%$, proporción de éxito de $50 \%$ y un margen de error de $9 \%$; con ella se aplicaron 115 cuestionarios a beneficiarios. ${ }^{3}$ Una vez establecida la muestra, se localizaron los estudiantes y se contactaron de manera personal; sin embargo, surgieron factores no controlables, como la deserción previa de los beneficiarios (baja definitiva o temporal), repetición de curso u otros. Así, de la muestra inicial (115), quedaron 51 (muestra definitiva); de modo que los beneficiarios finales se convirtieron en una muestra intencional. A ellos se les aplicó el cuestionario y se les solicitó información específica de 2017, dado que en ese año debían terminar el nivel medio superior.

La muestra intencional (o discrecional) es una técnica usada dadas las circunstancias de la investigación; aunado a ello, se usó el juicio propio para elegir a los beneficiarios. Por tanto, es un método de muestreo no probalístico que representa un ahorro de tiempo y dinero; su ventaja adicional es que las personas que participan en el estudio son limitadas y homogéneas (beneficiarios). Como toda muestra, entre las desventajas se encuentra la inadecuada representación de la población objetivo (Otzen y Manetrola, 2017). Frente a factores no controlables, se restablecen los criterios estadísticos de la nueva muestra: $81 \%$ en nivel de confianza (disminuye), con un error de $9 \%$ (se mantiente) y $50 \%$ de proporción de éxito (se mantiene).

\subsection{El cuestionario y la encuesta}

El cuestionario se estructuró con preguntas abiertas y cerradas, y se dividió en cinco secciones. En la primera parte se cuestionó sobre la familia del beneficiario y las condiciones de su vivienda. En la segunda parte, se obtuvo información sobre las características económicas de los estudiantes (ingresos mensuales de diversas fuentes). En la tercera parte, se averiguó su desempeño académico y su interés para continuar con su formación de capital humano en el nivel superior. En la cuarta

2 El muestreo aleatorio simple permite trabajar con una muestra probabilística, pues toda la población estudiada tiene las mismas probabilidades de ser elegida. Los resultados obtenidos se pueden inferir para toda la población. Tiene la ventaja de que, en caso de faltar una muestra, se puede usar otra con reemplazo o una sin reemplazo. Entre sus desventajas se encuentra que ocupan más tiempo y dinero para hacer este tipo de muestreo.

3 La base de datos proporcionó el nombre del beneficiario, escuela, nivel de estudios y horario de clases; de ésta se extrajo la muestra anterior 
parte, se indagó sobre los subsidios del gobierno que son otorgados al estudiante y cómo se usan para su formación educativa. En la última sección, se preguntó sobre las condiciones de la computadora portátil que les fue otorgada, incluyendo la frecuencia de uso y su estado físico.

\subsection{Procesamiento de la información recabada}

Toda la información recabada (cualitativa y cuantitativa) fue capturada en el programa Excel®; en las columnas se colocaron las preguntas realizadas y en las filas, las respuestas. Posteriormente, se construyeron gráficas descriptivas para visualizar los resultados.

De igual modo, con la información recabada, se hizo una distribución de ingresos de los beneficiarios para estimar su condición de pobreza; adicionalmente, para conocer la desigualdad económica entre los estudiantes, se construyó el índice de Gini. El índice de pobreza se calculó usando tres líneas de pobreza para zonas urbanas: pobreza extrema, con un valor de $\$ 1,325.56$ de ingreso mensual per cápita; pobreza de capacidades, con un valor de $1,625.80$, y pobreza patrimonial, con un valor de $\$ 2,659.61$ (Coneval, 2020a). Con base en esta clasificación, se analizó si el ingreso en el hogar puede ser un factor que propicie que los estudiantes logren, o no, concluir el nivel medio superior. Sobre el índice de Gini, se construyó una distribución de deciles usando los ingresos mensuales y se aplicó la ecuación para Gini $(\mathrm{G})=\left|1-\sum_{k=0}^{k=n-1}\left(X_{k+1}-K_{k}\right)\left(Y_{k+1}+Y_{k}\right)\right|$.

Donde $X=$ proporción acumulada de la población, $Y=$ proporción acumulada de ingresos, y $\mathrm{k}=$ decil. El resultado (en términos absolutos) mide la desigual distribución del ingreso; 0 es la máxima igualdad y 1 es la máxima desigualdad en la distribución.

Asimismo, con el programa Statistical Package for Social Sciences (SPSS $\left.{ }^{\circledR}\right)$ se realizó el análisis de correlación de Pearson para las siguientes variables: ingreso total y adeudos de asignaturas (para señalar si un mayor o menor ingreso afecta el rendimiento educativo para adeudar o no alguna asignatura cursada); horas recreativas y examen de admisión (para señalar si un mayor o menor tiempo dedicado a recreaciones afecta al estudiante en su decisión de continuar o no en su educación superior); horas educativas y examen de admisión (para indicar si un mayor o menor tiempo dedicado al estudio ocasiona que el estudiante tome la decisión de continuar o no con sus estudios de nivel superior); ingreso y examen de admisión (para indicar si un 
mayor o menor ingreso es determinante para que el estudiante tome la decisión de continuar o no estudiando). Los coeficientes obtenidos de las correlaciones anteriores sirven para conocer si existe una relación fuerte o débil entre las variables consideradas; es decir, si existe una dependencia total de una sobre otra (sentido directo o inverso), mientras que la significancia indica si ambas variables se asocian entre sí. Si el valor $\mathrm{p} \leq 0.05$ se rechaza, ambas relaciones no son significativas en 95 por ciento. Con este análisis se respondió la hipótesis planteada sobre si la utilización de la computadora portátil coadyuvó a culminar el nivel medio superior y permitió a los beneficiarios postularse al nivel superior.

\section{RESULTADOS DE LA INVESTIGACIÓN}

\subsection{Beneficiarios del programa Bienestar Digital (BD)}

De acuerdo con el INEGI (2014), durante el ciclo escolar 2014-2015 había 140 escuelas del nivel medio superior, 133 en el municipio de Mérida y siete en Umán; en estas escuelas había 4,326 beneficiarios del programa BD (Cuadro 1).

CUADRO 1

ESCUELAS Y ESTUDIANTES BENEFICIADOS DEL PROGRAMA BIENESTAR DIGITAL EN EL CICLO ESCOLAR 2014-2015

\begin{tabular}{ccc}
\hline Municipio & Total de escuelas & Total de estudiantes \\
Mérida & 23 & 4,174 \\
Umán & 7 & 152 \\
Total & $\mathbf{3 0}$ & $\mathbf{4 , 3 2 6}$ \\
\hline
\end{tabular}

Fuente: elaborado con datos del Gobierno del Estado de Yucatán (2018).

El programa BD se creó en Yucatán en 2013 bajo el gobierno de Rolando Zapata Bello (2012-2018); operó con base en las reglas de operación expedidas el 1 de febrero del 2013 a cargo de la Secretaría de Educación, mismas que en 2017 fueron modificadas. El programa consistió en entregar a los estudiantes beneficiarios de nivel medio superior una computadora portátil, a partir del primer año del nivel educativo de escuelas públicas del estado. Los solicitantes debían esperar la publicación de la convocatoria -emitida de manera anual en los primeros 15 
días del calendario escolar del ciclo lectivo- en el sitio del programa ${ }^{4}$ y cumplir con los requisitos que establecía la misma. Resalta la gran demanda de solicitudes, aunque los recursos presupuestales del programa eran limitados. Por lo tanto, para el criterio de elegibilidad, se estableció acreditar un cierto porcentaje de asistencia escolar y no adeudar asignatura alguna del nivel bachillerato.

\subsection{Caracterización socioeconómica de los beneficiarios del Programa Bienestar Digital}

Los beneficiarios promediaron 18.6 años de edad; 55\% contaban con 18 años; $16 \%, 19$ años; 14\%, 17 años, y en menor proporción, 23 y 28 años que representa $2 \%$, respectivamente. Los beneficiarios de entre 23 y 28 años son estudiantes adultos del nivel medio superior, bajo la modalidad denominada telebachillerato, que no necesariamente son recursadores o rezagados.

En relación con los integrantes de la vivienda donde habitan los beneficiarios, se sabe que los hogares tienen entre cuatro y cinco habitantes en promedio, y que las viviendas cuentan con diferentes servicios, entre los que la electricidad y el agua potable son los más importantes. Llama la atención que a pesar de que les otorgaron la computadora, sólo $29 \%$ de los hogares cuentan con servicio de internet y $18 \%$ tiene computadoras personales adquiridas de manera privada.

Otro aspecto importante en los hogares de los beneficiarios es el ingreso percibido. En este caso, en 59\% de los hogares, la aportación económica por trabajo remunerado le corresponde a una persona; $24 \%$, a dos personas, y 17\%, a tres o más personas. El ingreso promedio total aportado en cada hogar fue de $\$ 3,600.00$ al mes, y la mayor aportación proviene del padre o la madre, principalmente. En cuanto a la situación laboral de los beneficiarios, 78\% realizó algún trabajo remunerado, mientras que $18 \%$ no trabajó y $4 \%$ no especificó si trabajó; es decir, la mayoría tiene un trabajo y recibe un ingreso promedio mensual de $\$ 1,166.7$, que $54.2 \%$ destina para sus gastos escolares (transporte y alimentos, principalmente).

Por otra parte, se estimó el coeficiente de Gini sobre la distribución del ingreso entre los beneficiarios; el resultado fue 0.1692, lo

4 La página oficial fue dada de baja cuando la administración del gobierno estatal 2012-2018 concluyó. 
cual indica que se encuentra cerca del valor cero, es decir, cerca de la "perfecta igualdad". Según este resultado, la mayoría de los hogares de los beneficiarios tienen un ingreso mensual similar. Para complementar la condición social, los resultados de pobreza indican que estos hogares son considerados pobres, pero con diferentes tipos de carencia, pues $88 \%$ recae en la llamada "pobreza patrimonial", lo cual indica que la familia del beneficiario recibe un ingreso mensual de \$2,659.61 o más; mientras que $12 \%$ restante se encuentra en la "pobreza alimentaria", al recibir un ingreso mensual de $\$ 1,325.26$.

De este modo, la situación económica de los beneficiarios (pobreza con ingresos igualitarios) no parece ser suficiente para invertir en condiciones de mejoramiento del estudiante; el ingreso que reciben alcanza únicamente para adquirir productos de la canasta básica alimentaria, así como para cubrir gastos de salud, transporte, educación, entre otros. En repetidas ocasiones, el insuficiente ingreso provoca que muchos jóvenes no continúen con sus estudios, debido a que algunos deciden incorporarse al mercado laboral para subsistir junto con su familia.

\subsection{Características de los subsidios recibidos por los beneficiarios}

De acuerdo con los beneficiarios, $62 \%$ obtuvo alguna beca económica a través de los programas Prospera (federal), IBECEY (Instituto de Becas y Créditos Educativos del Estado de Yucatán) o por recursos propios de las escuelas, quienes en promedio les otorgaron mensualmente $\$ 1,000$, de los cuales $70 \%$ lo destinaron para gastos educativos. Cabe mencionar que, a pesar de que los subsidios fueron otorgados para incentivar a los estudiantes para mejorar su rendimiento educativo y para que concluyeran sus estudios, esto no se cumplió, debido a que algunos estudiantes adeudaron asignaturas, abandonaron la escuela o no continuaron estudiando. Sobre los adeudos, la mayoría de los beneficiarios no aprobaron, inicialmente, uno o hasta nueve cursos; solamente los estudiantes sobre el eje horizontal con valor cero lograron terminar el nivel medio superior sin problema académico.

\subsection{Uso de la computadora portátil según asignaturas cursadas}

Según la investigación, la computadora otorgada al beneficiario fue utilizada en su mayor tiempo para el estudio de las asignaturas corres- 
pondientes a los semestres escolares, en especial en los cursos de informática, ciencias de la salud y metodología de la investigación. Las horas de uso semanal se promediaron en cuatro; la menor frecuencia de uso osciló entre 8 y 10 horas en las asignaturas cursadas. De este modo, se hizo evidente que los beneficiarios usaron con menor frecuencia la computadora para las asignaturas de la escuela (excepto para ciertos cursos).

Por su parte, los programas informáticos que contribuyeron al desempeño del estudiante fueron principalmente: Word ${ }^{\circledR}$ y Excel ${ }^{\circledR}$, herramientas para realizar cálculos matemáticos, redactar ensayos o reportes de investigación, entre otras actividades.

\subsection{Problemáticas de las computadoras portátiles}

Aunque algunas computadoras funcionaron correctamente, otras presentaron anomalías, como fallas en el disco duro o memoria, o daños en el monitor o el teclado. En algunos casos, el sistema operativo no funcionó, y en otros, la máquina no permitió que se instalaran nuevos programas. Estos problemas se produjeron en 33\% de los beneficiados. El hecho de que las computadoras fueran ineficientes repercutió en el aprendizaje académico de los estudiantes.

A pesar de estas anomalías, la calificación que los estudiantes asignaron al programa promedió 7.8 puntos de un total de 10, lo cual puede ser considerado como aprobatorio. Entre las opiniones positivas, la mayoría indicó que las computadoras les sirvieron para realizar sus tareas, aumentar su desempeño académico y no gastar dinero para la renta de una. Entre los aspectos negativos, la minoría de los beneficiarios indicó que la computadora se descompuso, era lenta, no cargó satisfactoriamente programas necesarios o tenía memoria limitada. Igualmente, algunos mencionaron que en las escuelas no se podían conectar a internet.

\subsection{Caracterización educativa del estudiante beneficiario}

Los estudiantes entrevistados ${ }^{5}$ aprobaron sus cursos, lo que implica que pueden egresar del nivel medio superior en el tiempo establecido (tres años), según su modalidad oficial. Sin embargo, del total de encues-

51 estudiantes forman la muestra intencional. 
tados, $37 \%$ adeudó de dos a tres asignaturas por semestre; $22 \%$ reprobó alguna asignatura en el tercero y cuarto semestre, mientras que $13 \%$ reprobó algún curso del quinto semestre. ${ }^{6}$ Esto permite inferir que las computadoras entregadas no fueron suficientes para que los beneficiarios culminaran sus estudios sin tener problemas académicos. Por tal motivo, es importante conocer si el ingreso monetario de los hogares tiene una relación con que los estudiantes adeudaran alguna asignatura; para ello se utilizó una correlación lineal entre la variable "adeudo de asignaturas" e "ingreso total del hogar" (Cuadro 2).

\section{CUADRO 2}

Correlación entre "Adeudo de asignaturas" E "INGReso total del hogaR"

\begin{tabular}{cccc}
\hline & & Ingreso & Adeudo \\
\multirow{2}{*}{ Ingreso } & $\begin{array}{c}\text { Correlación de Pearson } \\
\text { (Significancia bilateral) }\end{array}$ & 1.0000 & -0.249 \\
\multirow{2}{*}{ Adeudo } & $\begin{array}{c}\text { Correlación de Pearson } \\
\text { (Significancia bilateral) }\end{array}$ & -0.249 & $(0.107)$ \\
\hline
\end{tabular}

Fuente: elaborado con el programa SPSS ${ }^{\circledR}$.

El resultado muestra una correlación negativa (o inversa) de - 0.249 entre ambas variables, lo que significa que cuando el ingreso total del hogar se incrementa, se espera que disminuya el adeudo de alguna asignatura. Esta afirmación es válida con un nivel de confianza cercano a $90 \%$; hay que recalcar que esta relación implica ingresos monetarios, no bienes físicos, como una computadora; no obstante, puede interpretarse que, si los beneficiarios tuvieran ingresos suficientes, podrían adquirir una computadora y mejorar su desempeño académico. ${ }^{7}$ Para complementar el resultado del Cuadro 2, la Gráfica 1 muestra la dispersión

6 Cabe recalcar que el sistema de bachillerato general consta de tres años y está divido en seis semestres. En este caso, los estudiantes de sexto semestre indicaron que no adeudaban alguna asignatura.

7 El Cuadro 2 muestra que la población considerada en la relación de ambas variables son 43 beneficiarios; es decir, 18.6\% menos de la muestra general. Esto se debe a que parte de los beneficiarios no indicaron el ingreso mensual que perciben en sus hogares; aunque se sabe que solamente un beneficiario adeudó cursos, mientras que los restantes salieron sin adeudos. De este modo, esta muestra representa $76 \%$ de confianza, con $9 \%$ de error muestral, $50 \%$ como proporción de éxito y con un valor " $z$ " de 1.175. Es importante aclarar que debido a que se utiliza una muestra intencional, el resultado es no probabilístico, pero los resultados aplican al grupo estudiado. 
de ambas variables, que confirma su relación negativa. Se observa que hay estudiantes que no adeudaron asignaturas y están entre los ingresos bajos; de igual modo, la gran mayoría de los que adeudaron pertenecen a aquellos que perciben ingresos bajos, relación que pudiera revertirse si los ingresos percibidos fueron medios o altos.

GRÁFICA 1

RELACIÓN ENTRE EL ADEUDO DE ASIGNATURAS E INGRESO TOTAL DEL TRABAJO

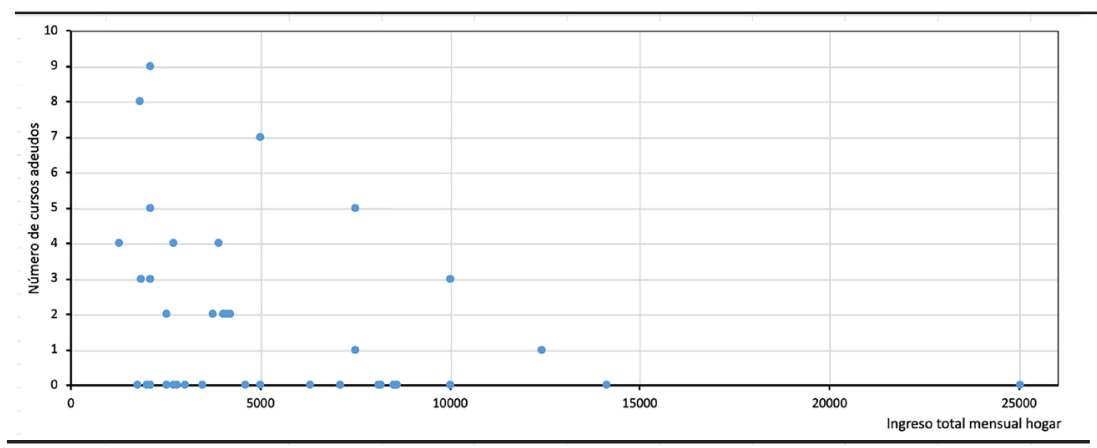

Fuente: elaborada con el programa SPSS ${ }^{\circledR}$.

Los resultados también indican que, al terminar el semestre de 2017, $65 \%$ de los beneficiarios optó por concluir su preparación académica; de modo que no intentaron aplicar al nivel superior; mientras que el porcentaje restante intentó ingresar al nivel superior dentro del sistema educativo público. Para el caso de los beneficiarios que declararon no continuar con su formación como capital humano, los motivos son diversos, pero el Cuadro 3 muestra que se debió a la falta de recursos económicos. Este motivo podría cambiar si el estudiante tuviera la seguridad de que si entra al nivel superior, contará con una beca, pues un bajo porcentaje de estudiantes no desea estudiar el nivel superior, aunque tuviera facilidades. 
El programa gubernamental Bienestar Digital... $\Gamma$ Ortiz-Pech, R.; W. M. Uc-May y R. Canto-Sáenz

CUADRO 3

RAZONES PARA NO CONTINUAR EL NIVEL SUPERIOR EN PORCENTAJE (\%)

\begin{tabular}{cccc}
\hline Problemática & $(\%)$ & Problemática & (\%) \\
\hline Bajo ingreso económico & 58 & Optó por trabajar & 6 \\
No quiso estudiar más & 9 & $\begin{array}{c}\text { Presentó problemas de } \\
\text { salud }\end{array}$ & 3 \\
\hline Tomó tiempo de descanso & 6 & $\begin{array}{c}\text { Se dedicó a labores } \\
\text { domésticas }\end{array}$ \\
\hline
\end{tabular}

Fuente: Uc (2019).

De los 51 beneficiarios, solamente 35\% intentó ingresar al nivel superior; $83 \%$ presentó su examen de admisión en escuelas públicas, y el restante, en instituciones privadas. Entre las escuelas públicas, 55.5\% lo intentó en la Universidad Autónoma de Yucatán (UADY), 11\% en el Instituto Tecnológico de Mérida, y 5.5\% en la Universidad Tecnológica Metropolitana (UTM). Entre las escuelas privadas se encuentran el Centro de Estudios Libres Universitarios (CLEU) y el Centro Educativo Rodríguez Tamayo (CERT), con 5.5\% para ambas. Este bajo porcentaje de estudiantes que decidieron continuar con sus estudios superiores muestra que el programa BD debió incentivar a los beneficiarios para que continuaran formándose como capital humano y para que contribuyeran con su vida productiva a la economía local y nacional, tal como postula la teoría endógena del crecimiento económico de Romer (1990) y Lucas (1988), quienes sostienen que la contribución del capital humano se transformará en una mayor productividad $\mathrm{y}$, por tanto, se espera tenga mejor percepción del ingreso personal; $\mathrm{y}$, de manera agregada, en incrementos del producto interno bruto de la economía.

\subsection{Uso de la computadora portátil y el examen de admisión al nivel superior.}

En este apartado se analiza cómo el uso de la computadora otorgada a los 51 beneficiarios de la muestra intencional se relaciona con la aplicación del examen de nivel superior que, como se describió anteriormente, un porcentaje bajo de estudiantes tomaron esa decisión. En primer lugar, $82 \%$ de los estudiantes beneficiarios dedicaron al menos cuatro horas semanales para usar la computadora para fines recreativos, 
mientras que $12 \%$ de los estudiantes destinó de 10 a 8 horas semanales para fines educativos. Estos resultados pueden ser interpretados como que no se dio un uso adecuado a la computadora para los fines establecidos y explican también el por qué de la reprobación de asignaturas. También se averiguó que un bajo porcentaje de estudiantes -menos de la mitad- usaron la computadora para fines educativos, para todas las categorías de horas de uso semanal.

Para complementar el argumento anterior, se realiza una correlación de Pearson entre las horas que la computadora fue utilizada para fines recreativos y los estudiantes que presentaron examen de admisión a nivel superior (universidad o tecnológico). El resultado presentado en el Cuadro 4 muestra un sentido positivo de 0.136; es decir, en la medida en que la computadora se usa más horas para fines recreativos, se espera que también los beneficiarios realicen el examen de admisión a nivel superior, lo cual es un resultado no esperado, ya que lo ideal es que se use más para fines educativos. Sin embargo, esto queda descartado por el nivel de significancia bilateral de 0.341 , lo que significa que ambas variables $^{8}$ no se asocian entre sí, por lo que esta correlación no se acepta.

\section{CUADRO 4}

CORRELACIÓN ENTRE EL USO DE COMPUTADORA PARA HORAS RECREATIVAS-EDUCATIVAS Y APLICACIÓN A EXAMEN SUPERIOR.

\begin{tabular}{|c|c|c|c|c|c|}
\hline & & Horas recreativas & $\begin{array}{l}\text { Aplicación de } \\
\text { examen }\end{array}$ & $\begin{array}{l}\text { Horas } \\
\text { educativas }\end{array}$ & $\begin{array}{l}\text { Aplicación de } \\
\text { examen }\end{array}$ \\
\hline Horas recreativas & $\begin{array}{l}\text { Correlación de Pearson } \\
\text { (Significancia bilateral) }\end{array}$ & 1.0000 & $\begin{array}{c}0.136 \\
(0.341)\end{array}$ & & \\
\hline $\begin{array}{c}\text { Aplicación de } \\
\text { examen }\end{array}$ & $\begin{array}{l}\text { Correlación de Pearson } \\
\text { (Significancia bilateral) }\end{array}$ & $\begin{array}{c}0.136 \\
(0.341)\end{array}$ & 1.0000 & & \\
\hline Horas educativas & $\begin{array}{l}\text { Correlación de Pearson } \\
\text { (Significancia bilateral) }\end{array}$ & & & 1.0000 & $\begin{array}{l}-0.018 \\
(0.902)\end{array}$ \\
\hline $\begin{array}{l}\text { Aplicación de } \\
\text { examen }\end{array}$ & $\begin{array}{l}\text { Correlación de Pearson } \\
\text { (Significancia bilateral) }\end{array}$ & & & $\begin{array}{l}-0.018 \\
(0.902)\end{array}$ & 1.0000 \\
\hline
\end{tabular}

Fuente: elaborado con el software SPSS ${ }^{\circledR}$.

8 Se usa la correlación de Pearson bajo el supuesto de que las variables se distribuyen de manera normal. Esta correlación, aunque no es significativa, se presenta como un resultado no esperado. En este sentido, la relación debió ser negativa; sin embargo, la evidencia empírica no permite aseverarlo. 
En contraste, el Cuadro 4 muestra los resultados de correlación de Pearson entre las horas educativas dedicadas al uso de la computadora y los que sí realizaron el examen de admisión para el nivel superior. La correlación entre ambas arrojó un coeficiente de -0.018; es decir, una relación negativa (inversa) bastante baja entre las variables. Si se destinan más horas a la semana al uso de la computadora para fines educativos, se esperaría que menos estudiantes realicen el examen de ingreso a nivel superior; un resultado no esperado. Por el nivel de significación de 0.902 , no se puede asegurar ese resultado, por lo cual no se puede concluir que las horas de uso de la computadora para fines educativos influyan en que el beneficiario aplique para continuar en niveles superiores; sin embargo, se presenta como evidencia empírica de la relación.

\subsection{Relación entre el ingreso del hogar y el examen de admisión al nivel superior}

Esta relación es interesante, pues postula que el ingreso del hogar del beneficiario influye en su decisión para realizar el examen de admisión a nivel superior. En este sentido, el resultado del Cuadro 5 muestra que el coeficiente de correlación entre ambas variables es 0.220 , lo cual indica que existe una correlación positiva (directa); es decir, conforme más nivel de ingresos monetarios posea el hogar, más beneficiarios aplicarán para el nivel superior, lo cual es algo esperado. Sin embargo, aunque existe una relación directa, esta correlación no es fuerte entre las variables "ingreso total del hogar" y "beneficiarios que presentaron el examen de admisión para ingresar al nivel superior"; por el nivel de significancia de 0.157 , no se puede asegurar esta relación en $95 \%$ de confianza.

Al relacionar este resultado con "adeudo de asignaturas" e "ingreso total del hogar" (Gráfica 2), se argumenta que el sentido esperado es adecuado, aunque la significancia resultó en $84 \%$ de confianza $;{ }^{9}$ el resultado se presenta para fines de evidencia empírica.

9 Aplica la limitante que, de los 51 beneficiarios, la submuestra para este resultado fue de 43 beneficiarios; esto se debe a que, como se mencionó, solamente estos estudiantes proporcionaron información sobre el ingreso monetario en sus hogares, de modo que la muestra restante no se contempló en el análisis de correlación. 
CUADRO 5

CORRELACIÓN ENTRE INGRESO MENSUAL DEL HOGAR Y EXAMEN DE ADMISIÓN

\begin{tabular}{cccc}
\hline & & Ingresos & Aplicación de examen \\
Ingresos & $\begin{array}{c}\text { Correlación de Pearson } \\
\text { (Significancia bilateral) }\end{array}$ & 1.0000 & 0.220 \\
Aplicación de \\
examen & $\begin{array}{c}\text { Correlación de Pearson } \\
\text { (Significancia bilateral) }\end{array}$ & $\begin{array}{c}0.220 \\
(0.157)\end{array}$ & 1.0000 \\
\hline
\end{tabular}

Fuente: elaborado con el software SPSS ${ }^{\circledR}$.

La Gráfica 2 muestra la relación binaria entre ellas mismas; cada punto identifica a un beneficiario. En este caso, los estudiantes que se encuentran en el eje 0 son los que deciden no continuar sus estudios de nivel superior, mientras que los que se hallan en el eje 1 son los beneficiarios que deciden continuar. En este sentido, las personas que perciben un ingreso mensual menor a $\$ 5,000$ son más propensas a dejar de estudiar. De igual modo, la gráfica indica que la mayoría de los beneficiarios se encuentran en el eje 0 , lo que advierte alta concentración de estudiantes con poca disposición para continuar estudiando. Asimismo, la gráfica muestra que el único ingreso mensual de $\$ 25,000$ es atípico y tiene la intención de continuar estudiando.

GRÁFICA 2

RELACIÓN ENTRE INGRESO DEL HOGAR Y EXAMEN DE ADMISIÓN

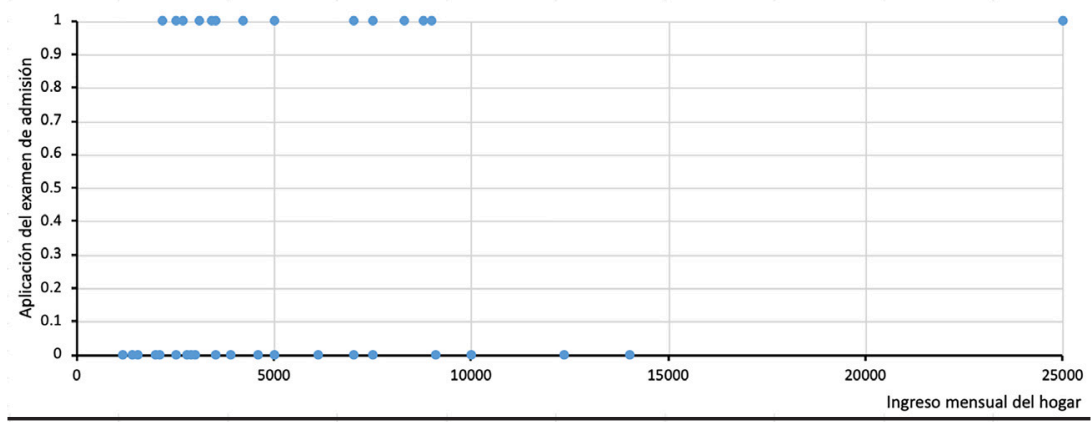

Fuente: elaborada con el programa SPSS ${ }^{\circledR}$. 
Este resultado puede explicarse porque el ingreso bajo de los hogares es uno de los problemas más comunes por el que los estudiantes no pueden continuar estudiando el nivel superior, ya sea en alguna universidad o instituto tecnológico. Por ello, el problema económico sigue siendo una limitante crucial para que los jóvenes de nivel medio superior continúen su formación educativa, debido a que algunos de ellos dejan la escuela para poder trabajar y generar un ingreso monetario para su hogar, a pesar de los subsidios o ayuda que el gobierno les otorga, incluyendo la computadora portátil.

En general, se concluye que estos resultados aplican solamente para los beneficiarios estudiados, ya que forman parte de una muestra intencional $\mathrm{y}$, por tanto, no pueden emplearse para la población total. No obstante, la evidencia empírica encontrada contribuye a entender el impacto de los programas sociales considerando los beneficiarios.

\section{CONCLUSIONES}

Los resultados obtenidos en este estudio concluyen que el programa gubernamental denominado Bienestar digital no cumplió con las expectativas de que los beneficiarios finalizaran el nivel medio superior; por tanto, la hipótesis planteada se rechaza. De 100\% de beneficiarios de la muestra inicial, alrededor de $56 \%$ no se encontraba en la escuela al momento del trabajo de investigación, ya sea porque ya no asistían a clases o porque habían reprobado. ${ }^{10} \mathrm{~A}$ pesar de que a los estudiantes les otorgaron la computadora y contaban con otros programas sociales, estos apoyos no fueron suficientes para continuar con sus estudios de nivel superior; el factor económico (ingreso monetario) fue la principal limitante.

Si bien el tamaño de la muestra fue limitante, es decir, fue menor a la planteada inicialmente, es necesario tomar reemplazos; sin embargo, de hacerlo, no se visualizaría la problemática del abandono escolar. Por tanto, los resultados y conclusiones de este trabajo aplican solamente para aquellos que formaron parte del estudio, ya que la muestra no es probalística. No obstante, estos hallazgos ayudan a entender la problemática de los programas sociales.

10 Por tanto, se consideran estudiantes rezagados, pues no cumplieron con el tiempo adecuado de egreso durante el periodo correspondiente. 
A pesar de ello, uno de los beneficios del estudiantado que recibió apoyo del programa Bienestar Digital está no haber pagado por la computadora, ya que su condición de pobreza les complicabla adquirirla en el mercado. Por tanto, el programa fue importante dentro de la vida educativa de los beneficiarios; sin embargo, es necesario que el programa mejore sus estrategias de intervención para evidenciar el impacto en la permanencia y conclusión en la formación en capital humano. Aquí el gobierno juega un papel crucial para que los estudiantes en desventaja tecnológica y pobreza no tengan obstáculos para cumplir con su derecho a la educación.

Los resultados permiten contrastar las políticas públicas. Este trabajo mostró que otorgar computadoras no tuvo un efecto significativo en el desempeño académico del estudiante, a pesar de que en tiempo de contingencia sanitaria, ${ }^{11}$ el uso de las computadoras para realizar investigaciones, tareas, cursos y otras actividades, se volvió necesaria. Estos sucesos abren la discusión sobre si es viable entregar computadoras a los estudiantes como apoyo gubernamental, a pesar de no cumplir con los objetivos de un programa social.

Por tanto, entre las recomendaciones obtenidas de este análisis se encuentran las siguientes: las computadoras otorgadas a los estudiantes en condición de pobreza deben tener un mínimo de calidad que contribuya a su desempeño escolar. Además, al terminar la vida útil del aparato, éste debe ser devuelto a un centro de acopio de residuos tecnológicos para colaborar con el cuidado del medio ambiente. También se recomienda que el beneficiario se comprometa a cumplir con los objetivos de cualquier programa social semejante al estudiado; de modo que, lo que enseña la teoría de Lucas (1988) o Romer (1990) sobre la formación de capital humano, pueda hacerse realidad; esto es, que los egresados de las escuelas contribuyan a la producción nacional o local para lograr un crecimiento económico.

11 Como la pandemia por Covid-19 que obligó al cierre de todo el sector educativo durante todo 2020, por lo que el uso de una computadora personal fue indispensable para continuar la formación educativa. 


\section{Bibliografía}

Aguilar, Jesús (2016), “TIC y políticas de equidad en el nivel medio superior mexicano: una panorámica", Innovación Educativa, 16 (72), Instituto Politécnico Nacional, México, pp. 133-158. <http://www.scielo.org.mx/ scielo.php?script=sci_arttext\&pid=S1665-26732016000300133> [10 de abril de 2020].

Bravo, Ernesto (2013), "El sector público en los modelos de crecimiento y desarrollo económicos: una aproximación al caso de la economía mexicana", Revista Economía informa, 379 (1), UNAM, México, pp. 3-22. <https:// doi.org/10.1016/S0185-0849(13)71312-4> [20 de junio de 2020].

Cardona, Marleny, Isabel Montes, Juan Vásquez, María Villegas y Tatiana Brito (2007), Capital humano: una mirada desde la educación y la experiencia laboral, Universidad EAFIT, Colombia. <http://publicaciones. eafit.edu.co/index.php/cuadernos-investigacion/article/view/1287/1166> [30 de abril de 2020].

Coneval (2020a), Medición de la pobreza. Evolución de las líneas de pobreza por ingreso. México. <https://www.coneval.org.mx/Medición/MP/Paginas/Lineas-de-bienestar-y-canasta-basica.aspx > [10 de marzo de 2020].

Coneval (2020b), Evaluación de la politica social. México. $<$ https://www.coneval.org.mx/Evaluacion/Paginas/Evaluaciones-y-resultados-de-programas.aspx $>$ [10 de diciembre de 2020].

Coneval (2020c), Monitoreo y evaluación de programas sociales. Yucatán. México. <https://www.coneval.org.mx/coordinacion/entidades/Yucatan/ Paginas/monyeval.aspx $>$ [10 de diciembre de 2020].

Congreso del Estado de Yucatán (2015), Constitución del Estado de Yucatán. <http://www.diputados.gob.mx/sedia/sia/spi/sapi-iss-01-16/Voces Const_Locales2015/YUCATAN\%202015.pdf $>$ [01 de marzo de 2020].

Díaz-Barriga, Ángel (2013), "TIC en el trabajo del aula. Impacto en la planeación didáctica", Revista Iberoamericana de Educación Superior Universia, 4 (10), UNAM, México, pp. 3-21. <http://www.scielo.org.mx/pdf/ ries/v4n10/v4n10a1.pdf $>$ [25 de mayo de 2020].

Galvez-Soriano, Óscar de Jesús (2020), "Could education increase the economic growth of Mexico?”, Revista Análisis Económico, 35 (89), UAM-Azcapotzalco, pp.37-64. <http://www.analisiseconomico.azc.uam.mx/index. $\mathrm{php} / \mathrm{rae} / \mathrm{article} / \mathrm{view} / 448>$ [29 de diciembre de 2020].

Gobierno del Estado de Yucatán (2013), Plan Estatal de Desarrollo de Yucatán 2012-2018. Consejo Estatal de Planeación de Yucatán. <http://www.yucatan.gob.mx/docs/transparencia/ped/2012_2018/PED_2012_2018.pdf> [10 de junio de 2020].

Gobierno del Estado de Yucatán (2017), “Acuerdo SEGEY 16/2017, Reglas de Operación del Programa Plataforma Educativa del Bachillerato, Bienestar 
Digital", Diario Oficia del Estado de Yucatán, 16 de marzo. <http://www. yucatan.gob.mx/docs/diario_oficial/diarios/2017/2017-07-05_2.pdf> [10 de marzo de 2020].

Gobierno del Estado de Yucatán (2018), Bienestar Digital. Transparencia y Rendición de Cuentas. Yucatán, México.

Instituto Nacional para la Evaluación de la Educación (INEE) (2011), La educación media superior en México, Informe 2010-2011. México. $<$ https:// historico.mejoredu.gob.mx/wp-content/uploads/2018/12/P1D235.pdf> [15 de abril de 2020].

INEE (2018), Panorama educativo estatal de la población indígena 2018. México. $\quad<$ https://www.inee.edu.mx/wp-content/uploads/2019/08/P3B115. pdf $>$ [27 de diciembre de 2020].

INEGI (2014), Anuario estadístico y geográfico de Yucatán 2014. Instituto Nacional de Estadística y Geografía. México. <https://www.inegi.org.mx/ app/biblioteca/ficha.html?upc=702825095116> [15 de mayo de 2020].

Lucas, Robert (1988), "On the mechanics of economic development", Journal of Monetary Economics, 22 (1), pp. 3-42. <https://doi.org/10.1016/03043932(88)90168-7> [5 de enero de 2021].

Mankiw, Gregory, David Romer y David Weil (1992), "A contribution to the empirics of economic growth", The Quarterly Journal of Economics, 107 (1), pp. 407-437. <https://eml.berkeley.edu/ dromer/papers/MRW QJE1992.pdf> [05 de junio de 2020].

Navarro, Iván (2005), “Capital humano: su definición y alcances en el desarrollo local y regional", Education Policy Analysis Archives/Archivos Analíticos de Políticas Públicas, 13 (1), Universidad del Sur de Florida, Estados Unidos, pp. 1-36. <http://www.redalyc.org/articulo.oa?id=275020513035> [12 de junio de 2020].

Ocegueda, Juan, Juan Meza y Domingo Coronado (2013), "Impacto de la educación en el crecimiento económico en México, 1990-2008", Revista Internacional Administración \& Finanzas, 6 (1), Institute for Business \& Finance Research, pp. 75-88. <https://ideas.repec.org/a/ibf/riafin/ v6y2013i1p75-88.html> [28 de diciembre de 2020].

OECD (2015), Students, computers and learning, making the connection. $<\mathrm{http}$ ://www.oecd.org/publications/students-computers-and-learning9789264239555-en.htm> [5 de enero de 2021].

Otzen, Tamara y Carlos Manterola (2017), "Técnicas de muestro sobre una población de estudio", International Journal of Morphology. 35 (1), Sociedad Chilena de Anatomía, Chile, pp. 227-232. <http://dx.doi.org/10.4067/ S0717-95022017000100037> [12 de junio de 2020]. 
Romer, Paul (1990), "Endogenous Technological Change”, Journal of Political Economy, 98 (5), The University of Chicago Press, EE.UU., pp. 71102. <https://web.stanford.edu/ klenow/Romer_1990.pdf $>$ [22 de junio de 2020].

Romer, D., G. Mankiw y D. Weil (1992). "A contribution to the empirics of economic growth", The Quarterly Journal of Economics, 107(2), pp. 407$437<$ https://doi.org/10.2307/2118477>

Secretaría de Gobernación (2008), "Programa Sectorial de Educación 20072012”, Diario Oficial de la Federación, 17 enero, México. <http://www. dof.gob.mx/nota detalle.php?codigo $=5028684 \&$ fecha $=17 / 01 / 2008>\quad[5$ de junio de 2020].

SEP (2012), Reporte de la Encuesta Nacional de Deserción en la Educación Media Superior. México. <http://www.sems.gob.mx/work/models/sems/ Resource/10787/1/images/Anexo_6Reporte_de_la_ENDEMS.pdf $>$ [5 de junio de 2020].

SEP (2013), Sistema Educativo de los Estados Unidos Mexicanos. Principales Cifras, Ciclo Escolar 2012-2013. México. <http://www.planeacion.sep. gob.mx/estadistica eindicadores.aspx $>$ [05 de agosto de 2020].

SEP (2014), Sistema Educativo de los Estados Unidos Mexicanos. Principales Cifras, Ciclo Escolar 2013-2014. México. <http://www.planeacion.sep. gob.mx/estadistica eindicadores.aspx $>$ [05 de agosto de 2020].

SEP (2015), Sistema Educativo de los Estados Unidos Mexicanos. Principales Cifras, Ciclo Escolar 2014-2015. México. <http://www.planeacion.sep. gob.mx/estadistica eindicadores.aspx $>$ [05 de agosto de 2020].

Uc, Wendy (2019), Impacto del programa Bienestar Digital en beneficiarios de escuelas de bachillerato de Mérida y Umán (2014-2015). Yucatán, México.

Unesco (2009), Medición de las tecnologías de la información y la comunicación (TIC) en educación-Manual del usuario. EE.UU. <https://www. cepal.org/socinfo/noticias/ documentosdetrabajo/0/40930/188309s.pdf $>$ [05 de enero de 2021].

Unesco (2015), La educación para todos, 2000-2015: logros y desafios. EE.UU. <http://unesdoc.unesco.org/images/0023/002325/232565s.pdf> [08 de septiembre de 2020].

Villareal, Edna (2016), "Externalidades del capital humano en México/Human capital externalities in Mexico", El Trimestre Económico, 83 (4), pp. 747788. <https://www.eltrimestreeconomico.com.mx/index.php/te/article/ view/238> [02 de diciembre de 2021]. 